\title{
Pratiques
}

Linguistique, littérature, didactique

169-170| 2016

Enseignement/apprentissage de la langue, des textes et des discours. 40\&nbspans de Pratiques

\section{L'orthographe : des systèmes aux usages}

Orthography: From Systems to Usages

Michel Fayol et Jean-Pierre Jaffré

\section{(2) OpenEdition \\ Journals}

Édition électronique

URL : http://journals.openedition.org/pratiques/2984

DOI : 10.4000/pratiques.2984

ISSN : 2425-2042

Éditeur

Centre de recherche sur les médiations (CREM)

Référence électronique

Michel Fayol et Jean-Pierre Jaffré, «L'orthographe : des systèmes aux usages », Pratiques [En ligne], 169-170 | 2016, mis en ligne le 30 juin 2016, consulté le 14 novembre 2019. URL : http://

journals.openedition.org/pratiques/2984; DOI : 10.4000/pratiques.2984

Ce document a été généré automatiquement le 14 novembre 2019.

(c) Tous droits réservés 


\section{L'orthographe : des systèmes aux usages}

Orthography: From Systems to Usages

Michel Fayol et Jean-Pierre Jaffré

1 Le $40^{\mathrm{e}}$ anniversaire de la revue Pratiques nous offre l'opportunité de revenir sur quatre décennies de travaux linguistiques et psycholinguistiques sur l'orthographe. Le hasard veut en effet que ce laps de temps corresponde à peu près aux changements, souvent importants, qui ont marqué ce domaine. Dans les limites d'un article, le propos restera forcément succinct, le but étant de rappeler au lecteur que ce parcours ne fut pas un long fleuve tranquille.

\section{Aspects linguistiques}

2 Les premières descriptions linguistiques de l'orthographe du français datent du début des années 70. Elles dominent les débats jusqu'à la seconde moitié des années 80 , date à laquelle la psycholinguistique, qui s'intéresse alors aux orthographes, en renouvelle durablement l'approche théorique.

\subsection{Linguistique et orthographe du français}

\subsubsection{Nina Catach et le plurisystème}

3 En France, il ne saurait être question d'orthographe sans citer celle qui a été à l'origine des tout premiers travaux sur le sujet. Dès 1973, dans la Recherche (1973a) puis dans Langue française (1973b), elle propose une analyse théorique popularisée sous le nom de plurisystème graphique. Cette terminologie est sans ambigüité: s'inspirant de l'approche structuraliste, et notamment des travaux sur la phonologie, N. Catach décrit l'orthographe du français comme une conjonction de systèmes à partir de laquelle elle élabore une définition complexe du graphème. Elle considère que notre écriture est formée de sous-systèmes dont les principaux dépendent de la correspondance avec les 
phonèmes (phonogrammes), avec la morphologie et avec le lexique (morphogrammes et logogrammes). À quoi s'ajoute un certain nombre de graphies sans justification synchronique, dont les liens avec le système actuel de la langue sont plus ou moins lâches (1973b).

4 Si l'option de N. Catach a pu être taxée de phonocentriste, c'est que les phonogrammes sont au cœur de son modèle tandis que les morphogrammes et les logogrammes n'en occupent que la périphérie. Une hiérarchie matérialisée par une série de cercles dont le centre est occupé par le code graphique proprement dit, c'est-à-dire le stock de graphèmes correspondant directement à nos phonèmes. Vient ensuite le cercle des morphogrammes ou graphèmes de morphèmes, la troisième zone, annexe celle-là, étant constituée par les logogrammes... (Catach, 1978). Les analyses ultérieures, publiées notamment dans Pratiques (Catach, 1979), ont sensiblement corrigé cette perspective, les morphogrammes y prenant une place plus importante. Ils deviennent en effet des signes qui complètent la notation des morphèmes et sont donc chargés d'une valeur sémique plus ou moins autonome (Catach, 1980). Mais finalement, comparée à la notion de phonème, celle de graphème n'en souffre pas moins d'un déséquilibre interstructurel dont ses trop nombreuses définitions sont le reflet (Pellat, 1988).

\subsubsection{Jacques Anis et la graphématique autonome}

5 Pour N. Catach, l'orthographe du français est donc d'abord une phonographie même si elle n'est pas que cela. Tel n'est pas le point de vue de J. Anis qui, à la fin des années 80 , veut traiter l'écrit comme un domaine autonome. De fait, la linguistique s'est interrogée très tôt sur le statut de l'écriture, le plus souvent pour l'exclure. Quelques linguistes - H. J. Uldall (1944), E. Pulgram (1951), L. Hjelmslev (1966) et surtout J. Vachek (1973), pour ne citer que ceux-là - ont toutefois préféré opter pour une voie médiane, établissant un parallèle entre l'écrit et l'oral. À cette paternité revendiquée, $\mathrm{J}$. Anis ajoute - avec le recul on peut dire non sans risques - des théories modernes de la lecture qui établissent une relation directe entre unités graphiques et signifiés, sans oralisation. Un postulat invalidé depuis.

6 L'écrit - et donc l'orthographe - peut-il constituer une norme linguistique indépendante de l'oral ? Telle est l'option choisie par J. Anis (1983). Il veut en effet rendre compte de la graphie d'une langue sans référence à la phonie, l'écrit n'étant plus un code secondaire transcrivant la langue stricto sensu, qui est orale, mais une part intégrante du système linguistique. Pour autant, J. Anis ne se prive pas d'un parallèle structurel avec l'oral puisqu'il s'inspire de l'opposition entre voyelles et consonnes pour distinguer deux classes de graphèmes - les nodes, ou noyaux syllabiques, et les sates, leur satellite. Cette approche autonomiste a certes eu le mérite de susciter la réflexion sur le statut de l'écrit et sur ses relations avec l'oral. En revanche, comparée au plurisystème de N. Catach, elle n'a que très peu influencé le regard que la société portait sur son orthographe. Ses limites épistémologiques ont même été évoquées quelques années plus tard, lors d'une table ronde sur l'autonomie de l'écriture (Puech \& Anis, 1997). 


\subsubsection{B. Cerquiglini et la diachronie}

7 Dans les années 90, le champ de l'orthographe française a bénéficié d'un apport nouveau avec l'irruption d'une conception dynamique de la genèse orthographique. Dans un ouvrage paru en 1996, B. Cerquiglini propose en effet une description diachronique de la formation de l'orthographe du français. Ce faisant, il décrit l'émergence d'un principe qu'il nomme graphique et qui met à distance le principe phonologique, Il démontre comment l'orthographe se constitue en une sémiologie à part entière, qui se sert de l'étymologie pour mieux servir le lecteur. La graphie reste bien une représentation linguistique mais elle transcrit moins des sons qu'une image de la langue. L'orthographe moderne nait par conséquent d'une graphie de l'ancien français qui est tout à la fois au service de l'œil et de l'oreille. Cette genèse historique de l'orthographe accorde donc un droit de cité à la part visuographique. Sans nier l'importance de la base phonographique, elle explique comment celle-ci évolue et peut se transformer pour mieux servir les fonctions que l'écrit est amené à remplir au fur et à mesure que la demande sociale évolue. Moins statique que le plurisystème de N. Catach ou que la graphématique autonome de J. Anis, le point de vue sémiologique de $\mathrm{B}$. Cerquiglini privilégie une véritable dynamique de l'orthographe.

Une telle approche suggère de facto la dualité de l'orthographe. La phonographie, base indispensable à l'économie du système, n'est finalement qu'une condition nécessaire de l'orthographe. Pour remplir les fonctions qu'une société assigne à l'écrit, et qui peuvent se modifier avec le temps, celui-ci doit aussi tenir compte de critères visuels, de façon à proposer des images graphiques qui permettent au lecteur expert de traiter plus aisément l'information. Preuve qu'une orthographe n'est pas seulement tributaire de la phonologie d'une langue mais également du signe linguistique tel que le définissait $\mathrm{F}$. de Saussure (1972). Et l'histoire fournit de ce point de vue une excellente source d'information sur les changements qu'une orthographe doit supporter pour y parvenir. C'est ce que montrent les travaux sur la genèse des blancs graphiques qui décrivent l'émergence d'une iconicisation de l'écrit capable de favoriser une meilleure saisie visuelle (Saenger, 1982, 1989).

\subsection{Linguistique et orthographes du monde}

9 Au cours des années 80, les travaux sur l'orthographe ont pris un nouveau tournant avec la parution d'études sur la litéracie, c'est-à-dire la façon dont les jeunes enfants apprennent à lire et à écrire dans une orthographe donnée (Jaffré \& Reuter, 1985). Dans leur grande majorité, les ouvrages ou les articles rendant compte de ces travaux étaient écrits en anglais, ce qui a retardé leur diffusion en France. Parmi les très nombreuses publications sur cette question, on peut citer, de façon sélective, et sans tenir compte des écoles de pensée ou du thème traité - lecture ou orthographe -, les ouvrages d'U. Frith (1980), de L. Henderson (1984), de S. Templeton \& D. Bear (1992), ou encore de C. a. Perfetti, L. Rieben \& M. Fayol (1997).

\subsubsection{L'apport de la litéracie}

10 D'une façon très générale, ces travaux nous ont appris que les processus d'acquisition d'une orthographe, quelle qu'elle soit, présentent une forme d'universalité. La phonographie en constitue le principe d'économie indispensable à tout système 
graphique. Pour apprendre à lire et à écrire, il faut être capable de percer le code et de maitriser un ensemble d'unités récurrentes, qu'elles correspondent à des phonèmes ou à des syllabes. Cette condition sine qua non peut certes prendre des formes variables mais sa nécessité est telle que l'acquisition d'orthographes qui semblent à priori échapper à cette contrainte nécessite des unités de transition. Les premiers apprentissages des caractères chinois recourent ainsi à l'outil alphabétique qu'est le pinyin (voir par exemple Ho \& Bryant, 1997).

11 Mais la maitrise de la dimension phonographique, indispensable lors des premiers apprentissages, ne suffit pas. Une orthographe doit également prendre en charge la dimension sémiographique, née de la représentation de la morphologie et du lexique propres à une langue. De ce point de vue, une bonne orthographe doit être capable de donner à voir le sens linguistique quitte, pour y parvenir, à compter sur l'aide objective de la diachronie pour transformer la phonographie. On se rend compte en effet que des orthographes construites initialement sur une phonographie plutôt fidèle biunivoque - font avec le temps des concessions au sens. C'est en français le cas du « $s$ » du pluriel, phonogramme tombé en désuétude puis remotivé à des fins morphographiques.

\subsubsection{Une linguistique comparative}

12 Les travaux sur la litéracie ont finalement ouvert la voie à des entreprises linguistiques plus nettement comparatives. C'est par ce biais que des orthographes différentes de celle $d u$ français ont été prises en compte, les modalités de l'acquisition posant en creux la question de la structure orthographique. Plusieurs ouvrages ont alors offert, selon des points de vue théoriques pouvant différer sensiblement, une description comparée des principales orthographes du monde (DeFrancis, 1989; Sampson, 1985 ; Coulmas, 1989; sans oublier l'extraordinaire somme de Daniels \& Bright, 1996). Des approches moins généralistes ont elles aussi apporté leur pierre à l'édifice de la linguistique de l'écrit - J. DeFrancis (1984) sur le chinois, Y.-K. Kim-Renaud (1997) sur le coréen, ou encore C. Seeley (1991) sur le japonais, etc.

\subsubsection{Une approche englobante}

13 La mise en perspective de ces analyses a permis d'imaginer un modèle linguistique à la fois simple et productif qui intègre l'ensemble des orthographes du monde, celle du français y compris. Ainsi, au-delà des spécificités qui peuvent caractériser chacune d'entre elles, une orthographe est par définition un compromis interactif entre une phonographie et une sémiographie (Jaffré, 2003). La phonographie, base systémique, se compose d'unités graphiques qui peuvent correspondre à des phonèmes, comme dans l'ensemble des orthographes d'Europe, ou des syllabes comme c'est le cas au Japon et en Chine. La sémiographie subsume la phonographie et regroupe l'ensemble des formes graphiques significatives, selon des modalités certes variables mais qui, dans tous les cas, remplissent la même fonction. En Occident, les orthographes utilisent abondamment les blancs graphiques pour isoler des formes visuographiques accessibles; en revanche, des orthographes comme celle du chinois ${ }^{1}$, associe des morphèmes et des caractères, un mot pouvant correspondre à deux ou trois caractères. Cette approche, que schématise le tableau ci-dessous, est détaillée dans les chapitres linguistiques de M. Fayol \& J.-P. Jaffré $(2008,2014)$. 
Tableau 1. Typologie orthographique

\begin{tabular}{|l|l|l|}
\hline \multirow{2}{*}{ SÉMIOGRAPHIE } & \multicolumn{2}{|l|}{ PHONOGRAPHIE } \\
\cline { 2 - 3 } & alphabets & syllabogrammes \\
\hline $\begin{array}{l}\text { Morphogrammes } \\
\text { (morphèmes) }\end{array}$ & $\begin{array}{l}\text { écritures morphoalphabétiques } \\
\text { (ex. : hangul coréen) }\end{array}$ & $\begin{array}{l}\text { écritures morphosyllabiques } \\
\text { (ex. : chinois) }\end{array}$ \\
\hline $\begin{array}{l}\text { Logogrammes } \\
\text { (mots) }\end{array}$ & $\begin{array}{l}\text { écritures logoalphabétiques } \\
\text { (ex. : français, arabe) }\end{array}$ & $\begin{array}{l}\text { écritures logosyllabiques } \\
\text { (ex. : japonais) }\end{array}$ \\
\hline
\end{tabular}

\subsection{Vers une sociolinguistique de l'orthographe}

14 Au cours de ces dernières années, les analyses théoriques sur l'orthographe se sont stabilisées, le point de vue binaire présenté ci-dessus étant adopté par le plus grand nombre. On assiste en revanche au développement de travaux sociolinguistiques qui s'interrogent sur les raisons pour lesquelles les orthographes, les plus anciennes surtout, suscitent un conservatisme tel que des réformes, pourtant bien utiles, provoquent des réactions souvent négatives. C'est évidemment le cas en France (Baddeley et al., 2013)2 , en Allemagne (Johnson, 2005), et dans bien d'autres pays.

L'autre source d'intérêt sociolinguistique vient des langues sans tradition écrite qui désirent se doter d'une orthographe. Comment se fait-il en effet que les futurs usagers jugent fréquemment trop techniques les solutions que leur proposent les linguistes? Une telle attitude confirme que les orthographes sont des outils sociologiquement complexes, sensibles à des facteurs - historiques, identitaires, ethniques, culturels et politiques - qui dépassent de très loin le champ de la linguistique (Sebba, 2007). Les orthographes dépendent en fait d'un usage social, pour ne pas dire communautariste, qui conditionne leur dimension iconique, à savoir les effets que produit l'image graphique sur les usagers. Ainsi, et pour ne citer que ce cas, au moment de créer la nouvelle orthographe du serbe, V. Karadzic tint absolument à y introduire la lettre « $j$ ", perçue comme la marque du catholicisme (Sebba, 2009).

Le regard rétroactif sur les orthographes révèle, par comparaison, le déficit d'études dont souffre aujourd'hui l'orthographe du français, tout spécialement dans le domaine de la sociolinguistique. Comment expliquer par exemple que tant de Français s'entêtent encore et toujours à refuser des aménagements orthographiques qui, pourtant, allègeraient leur tâche de scripteur? Pour ne rien dire de tous ces adultes qui trouvent un tel de plaisir dans l'exercice d'une dictée qui leur causa tellement de tracas lorsqu'ils étaient élèves.

17 Si la perspective sociolinguistique de l'orthographe demeure encore largement en chantier, l'approche psycholinguistique a fait en revanche l'objet de très nombreuses recherches. En voici un bref panorama. 


\section{Aspects psycholinguistiques}

18 Les recherches des psychologues portant sur l'orthographe en production sont relativement récentes. Elles suivent en général avec retard celles qui abordent la lecture et son apprentissage, et donc l'orthographe en perception et, éventuellement, en compréhension. Elles restent dans l'ensemble plus rares, moins théorisées et moins sophistiquées. Ainsi, le rapport du National Reading Panel (2000) n'évoque ni la production orthographique, ni la rédaction de textes, laissant sans bilan toutes les activités de production écrite. Ainsi encore, P. H. Seymour, M. Aro \& J. M. Erskine (2003) comparent les acquisitions d'élèves de première et deuxième années de scolarité dans différents systèmes orthographiques, certains plutôt transparents (finnois, italien, espagnol, allemand), d'autres plutôt opaques du fait de l'existence d'inconsistances dans les correspondances et d'influences morphologiques (portugais, danois, français, anglais). Ils mettent en évidence que la reconnaissance des mots simples et du décodage est plus rapide et efficiente avec certains systèmes (espagnol, italien) qu'avec d'autres, notamment portugais, français, et plus encore anglais. Toutefois, la comparaison ne porte que sur la lecture. Il faut attendre les travaux de M. Caravolas (2004; Caravolas et al., 2013) pour voir s'amorcer des études équivalentes en production orthographique, mais sur un nombre plus limité de systèmes (anglais, espagnol, croate, tchèque) et chez des élèves suivis pendant plusieurs mois. Ces recherches posent le problème de la diversité des systèmes orthographiques et de l'impact de ceux-ci sur l'apprentissage (Joshi \& Aaron, 2006). Les difficultés rencontrées se révèlent plus complexes à surmonter que dans le cas de la lecture, d'abord en raison de la moindre consistance des associations entre phonèmes et lettres (ou graphèmes) dans le sens de la production et ensuite, du fait qu'intervient une composante motrice.

Il est possible de tracer une évolution des thèmes. Ceux-ci trouvent largement leur inspiration dans les données rapportées par l'étude des troubles. Ce sont les neuropsychologues qui ont élaboré les premiers modèles dits à double voie, ce sont eux encore qui ont apporté les premières observations empiriques, ce sont eux également qui ont mis en évidence le rôle de la phonologie. Les modèles les plus actuels restent issus des recherches en neuropsychologie (Rapp \& Lipka, 2010; Tainturier \& Rapp, 2001) et se trouvent complétés par des tentatives de simulations (modèles dits computationnels ; Brown \& Loosemore, 1994), l'articulation entre les deux approches restant encore problématique. Ces modèles et ces données ont inspiré les recherches des psychologues s'attachant à l'étude de l'apprentissage. De manière un peu caricaturale, il est possible de distinguer, en parallèle avec la trame proposée ci-dessus par J.-P. Jaffré, deux grandes périodes : celle du «tout-phonologique » puis celle de la recherche d'une éventuelle autonomie de l'écrit.

\subsection{La dominance de la phonologie}

20 Une large série de résultats, dont la proportion s'accroit au fil des décennies, établit à partir des années 80 l'importance de la phonologie pour l'apprentissage de la lecture et de l'écriture. Les arguments émanent d'abord d'études de cas rapportées par des neuropsychologues. Ils proviennent ensuite de recherches établissant des corrélations entre les performances en lecture-écriture et les performances en conscience phonologique, établies à travers des épreuves diverses de segmentation, soustraction, 
adjonction, fusion, etc. de segments linguistiques de tailles variables (syllabes, rimes, phonèmes): de manière générale, qu'il s'agisse d'approches transversales (enfants d'âges ou de niveaux scolaires différents) ou longitudinales (élèves suivis pendant des périodes plus ou moins longues), les données attestent l'existence d'une relation significative et forte entre le degré de réussite aux épreuves de conscience phonologique et les scores aux tests de lecture, et plus encore de production orthographique (lorsque de tels tests sont proposés, ce qui reste relativement rare), cela d'autant plus que les systèmes orthographiques sont consistants (Caravolas, 2004 ; Ziegler et al., 2010). Les corrélations élevées suggèrent la possibilité d'une relation causale allant des premières aux seconds (lecture et écriture). Cette relation a été testée en lecture et a permis de confirmer par des entrainements parfois étalés sur le long terme le rôle déterminant de la conscience phonologique pour l'apprentissage de la lecture chez des élèves en grande difficulté (Torgesen, 2005). À notre connaissance, il n'existe pas d'équivalent pour ce qui concerne la production orthographique.

Les recherches conduites auprès d'enfants ordinaires et portant sur l'apprentissage de la production orthographique ont été essentiellement développées à partir des travaux de D. Share $(1995,2004)$. Dans les langues à écriture alphabétique, l'apprentissage de l'écrit nécessite la découverte que les séquences de lettres (ou de graphèmes) entretiennent des correspondances régulières - parfois complexes - avec les séquences sonores (de phonèmes), ce qui correspond au principe alphabétique. Progressivement, du fait de la pratique et de l'instruction, l'enfant met en place une procédure de déchiffrage faisant correspondre en lecture à haute voix les configurations sonores aux suites de lettres et une procédure de production lettre à lettre (en écriture) de toutes les configurations sonores reposant sur la médiation phonologique. Ces deux procédures sont dites génératives car elles permettent de lire et de transcrire toutes les configurations respectivement écrites et sonores jusqu'alors non rencontrées, même si elles conduisent à des lectures ou productions orthographiques non conventionnelles (Sprenger-Charolles et al., 2003). L'apprentissage guidé de la phonographie et la pratique de la lecture et de l'écriture ont trois effets. Premièrement, ils entrainent l'extension de la connaissance des phonèmes et des associations qu'ils entretiennent avec les graphèmes. Deuxièmement, comme le montrent les données rapportées par $\mathrm{D}$. Share $(1995,2004,2008)$, le déchiffrage des mots induit au moins partiellement leur mémorisation, de sorte qu'ils vont constituer un lexique orthographique permettant, comme le décrit le modèle à double voie, la récupération directe des items fréquents, en lecture comme en écriture (Martinet et al., 2004). Troisièmement, le déchiffrage encore conduit à la mémorisation de séquences de lettres fréquemment associées (des chunks : bigrammes, trigrammes, mais aussi suites du type -iste, -anque, etc.), séquences qui facilitent la lecture et la transcription d'items nouveaux. Selon la thèse de $\mathrm{D}$. Share, tout décodage réussi (et donc toute médiation phonologique) aboutirait à la constitution en mémoire d'une trace lexicale ou sous-lexicale stable. Les épreuves utilisées pour tester ces traces consistent soit en relecture (devenue plus rapide), soit en reconnaissance parmi des distracteurs soit en production orthographique sous dictée. Or, deux faits ressortent dans ce dernier cas, notamment pour ce qui a trait aux systèmes orthographiques peu consistants (néerlandais, anglais, français). D'une part, les performances restent relativement faibles; d'autre part, la qualité du décodage graphophonologique n'est que modestement corrélée à l'exactitude des productions. D'autres facteurs interviennent qui permettent de rendre compte des performances, notamment les performances antérieures en orthographe ${ }^{3}$. 


\subsection{Le poids de la dimension orthographique} rappellent que, si la lecture dans les systèmes alphabétiques s'enracine dans le langage oral et la phonologie, elle a également une dimension visuelle (voir aussi Dehaene, Cohen, Morais, \& Kolinsky, 2015). Elle s'appuie notamment chez les experts sur un traitement en parallèle des mots, de sorte que l'identité des lettres et leurs positions soient prises en compte. La lecture s'apparente plutôt à une épreuve de reconnaissance des formes orthographiques et tolère des traitements flexibles portant sur des items dont les configurations peuvent dévier des formes conventionnelles. Par exemple, un texte reste lisible rapidement lorsque les mots qui le constituent présentent des inversions des lettres internes (retse au lieu de reste par exemple) mais conservent les identités et positions des lettres extrêmes (Lété \& Fayol, 2013). Il n'en va pas de même de la production orthographique. Cette dernière exige que les identités et les positions relatives des lettres ou des graphèmes soient respectées. Ces contraintes ont pour corollaire que les formes orthographiques des mots présentent un degré élevé de précision. Dans un système orthographique comme celui du français, ces contraintes soulèvent d'importants problèmes d'apprentissage.

Les possibilités de transcription des différents phonèmes du français sont dans certains cas relativement nombreuses (en tout, 130 graphèmes pour 32 à 36 phonèmes). Des lettres sans correspondants phonologiques apparaissent souvent. Par exemple, certaines consonnes sont doublées sans opposition phonologique comme dans addition (qui serait prononcé à l'identique si la transcription était adition). Beaucoup de mots se terminent par des consonnes muettes (foulard) dont certains renvoient à la morphologie dérivationnelle (grand $\rightarrow$ grande). Enfin, la morphologie flexionnelle comporte des marques qui n'existent qu'à l'écrit, comme le -s du pluriel nominal ou le $n t$ du pluriel verbal (Dubois, 1965).

Ces spécificités du français nous ont amenés à rechercher si les traitements orthographiques ne pouvaient pas relever d'une autonomie de l'écrit, c'est-à-dire d'une relative indépendance par rapport à la phonologie (Bonin, Pacton \& Fayol, 2001). Des arguments issus des études neuropsychologiques, des travaux expérimentaux en production militent en faveur de cette conception. Ils expliquent en quoi la phonologie, indispensable pour mettre en place les tout-débuts de la lecture et de l'écriture, ne suffit ni pour assurer l'apprentissage et l'utilisation du lexique orthographique, ni pour rendre compte des phénomènes d'accord en genre et en nombre pour lesquels des marques écrites doivent être mobilisées, y compris par des adultes, sans pouvoir disposer d'indices phonologiques.

\subsubsection{Les régularités graphotactiques}

Certaines configurations de lettres n'ont pas de correspondants phonologiques ( $-h$ - ou encore les doubles consonnes) ou leurs variations (par exemple $o$, au, eau) ne sont pas associées à de telles correspondances. Les rédacteurs ne peuvent donc s'appuyer sur leur connaissance de l'oral pour les produire. Ainsi les doubles consonnes (DC) constituent une source d'erreurs dans les productions usuelles (Lucci \& Millet, 1994). Une question intéressante consiste à s'interroger sur l'apprentissage de telles spécificités orthographiques. S. Pacton et ses collègues ont conduit une large série de 
recherches qui ont mis en évidence que l'appréhension des DC est très précoce et précise : par exemple, très tôt dans la scolarité les enfants détectent que les consonnes sont doublées mais non les voyelles, que certaines consonnes ne sont pas ou très rarement doublées $(v, k)$ alors que d'autres le sont fréquemment $(t, l, m)$ (Pacton et al., 2001). Leurs performances ne dépendent pas des associations entre présence de DC et changements vocaliques (jeter versus jette; Pacton \& Fayol, 2000), ce qui est retrouvé en finnois dès avant que les enfants ne sachent associer les DC à leurs correspondances phonologiques. Point fondamental: ces régularités propres à l'orthographe sont apprises implicitement, par la pratique de la lecture et de l'écriture, sans avoir donné lieu à un enseignement. La précision de ces acquis étonne: les enfants répondent précocement que opécile est un item plus probablement français que eaupécile. En somme, ils s'avèrent sensibles à la distribution statistique des voyelles et des consonnes (de leurs identités et de leurs positions) dans les formes orthographiques sans que celle-ci leur ait été enseignée. Cette sensibilité précoce facilite souvent le décodage et les transcriptions; il arrive néanmoins qu'elle gêne la mémorisation d'une forme lorsque celle-ci comporte des suites de lettres ne suivant pas ces régularités (Fayol, Sahli \& Souny-Benchimol, 2015 ; Pacton, Fayol \& Lété, 2008). Ces derniers nécessitent alors de recourir à une instruction explicite (Fayol, Grimaud, \& Jacquier, 2013) ou à la mobilisation d'un enseignement portant sur des règles de morphologie dérivationnelle. Elle contrarie même l'application des règles morphologiques, par exemple la transcription des diminutifs en -eau (Pacton, Fayol \& Perruchet, 2005) - par exemple concernant les diminutifs en -eau ou en -ette) (Fayol et al., en préparation).

\subsubsection{Les phénomènes d'accord}

En français écrit, les marques du genre et du nombre (et beaucoup d'autres) doivent être marquées par adjonction de $-e$ au féminin, de $-s$ au pluriel pour les noms et les adjectifs, par ajout de -nt au pluriel pour les verbes. Là encore, ces marques n'ont qu'exceptionnellement des correspondants phonologiques (dans le cas des liaisons). Se pose donc une série de questions : comment les enfants découvrent-ils et interprètentils ces marques? Comment les activent-ils lors de la production puisqu'aucun indice audible n'est disponible? Plusieurs séries de recherches ont été conduites, qui montrent que les enfants découvrent et interprètent très vite ces marques mais omettent longtemps de les ajouter à la fin des mots : ils y parviennent d'abord avec les noms, puis les adjectifs (mais dans certaines limites) et les verbes (Fayol et al., 1999; Totereau, Barrouillet \& Fayol, 1998). L'apprentissage s'effectue par le biais d'un enseignement explicite, dispensé dès le CE1 et qui aboutit à une relative maitrise en fin de CE2 pour les cas les plus simples (Thévenin et al., 1999). Le recours rapide et systématique à ces marques est en place dès le cours moyen (Fayol, Hupet \& Largy, 1999) mais il implique que les élèves comme les adultes mobilisent de l'attention du fait de l'absence d'indice phonologique et gèrent en mémoire la distance entre les items intervenant dans l'accord. Ainsi, dans un article du journal Le Monde (04/02/2016, p. 4), on trouve: "Sur des équipements tels des générateurs de vapeur très faiblement radioactif...", l'accord de radioactif (avec générateurs) n'est pas effectué vraisemblablement en raison de la distance qui sépare les deux termes. De manière générale, il suffit que l'attention soit divertie ou que son niveau baisse pour voir apparaitre des erreurs : omissions ou substitutions de marques. Ces dernières résultent soit de l'attraction par un item proche (le chien des voisins arrivent; Fayol, Largy \& 
Lemaire, 1994) soit de la remémoration automatique d'un homophone plus fréquent (le facteurs a des lettres et il les timbres: timbres comme nom est plus fréquent que timbre ou timbrent comme verbe; Largy, Fayol \& Lemaire, 1996). Ces exemples illustrent les difficultés auxquelles se trouvent confrontés les francophones : même les meilleurs auteurs sont victimes de telle erreurs, non qu'ils ignorent les règles, non qu'ils ne sachent pas les appliquer, mais plus simplement parce que la vigilance requise leur fait défaut à un moment ou à un autre. Et la relecture, souvent efficace pour détecter et corriger les erreurs est elle-même parfois prise en défaut. En d'autres termes, la gestion des phénomènes d'accord soulève en français des problèmes tenant à l'homophonie et oblige les rédacteurs à faire preuve de vigilance pour prévenir les omissions ou substitutions de marques. Cela vaut pour les accords des noms, des adjectifs et des verbes. Cela vaut aussi pour les confusions entre infinitif et participe passé comme entre adverbe et participe présent (Pacton \& Fayol, 2003).

\subsection{La nécessaire prise en compte des interventions}

La plupart des travaux publiés dans le champ des études portant sur l'orthographe, en perception comme en production, s'appuient sur des données qui postulent (implicitement) que les données recueillies reflètent des processus stables. Il s'agit d'une étape nécessaire dans les approches scientifiques ; mais elle n'est pas suffisante. Les performances orthographiques des individus, enfants comme adultes évoluent, le plus souvent à long terme mais aussi parfois à très court terme sous l'influence de deux grands facteurs. Le premier, difficile à maitriser, mais dont les effets se manifestent à l'observation des corpus de productions tient à l'exposition à des formes orthographiques dont les caractéristiques sous-lexicales changent. Ainsi, les étudiants de sciences humaines confrontés de manière croissante aux textes rédigés en anglais tendent de plus en plus au fil des années universitaires à transcrire "connection » au lieu de connexion. Ainsi encore, les enseignants ayant à corriger des copies en grand nombre rencontrent parfois plusieurs dizaines de fois les mêmes erreurs orthographiques (par exemple "accomoder" au lieu de accommoder). Ils en arrivent alors à douter de leur propre connaissance! Il s'agit là d'effets consécutifs à un apprentissage implicite associé à des fréquences croissantes d'exposition. Ces effets ont été étudiés et leur impact évalué (Fayol \& Jaffré, 2008), mais il est impossible de s'en préserver du fait de l'usage de plus en plus fréquent des courriels. De fait, on peut s'attendre à ce que l'écrit, longtemps associé aux pratiques scolaires contraignant très fortement les usagers à respecter les formes conventionnelles se remette à évoluer (comme il l'a fait jadis; voir Chervel, 2006) et qu'en conséquence des régularisations se mettent en place, par exemple en doublant systématiquement le -n- dans la configuration -onne. Il parait probable que cette modification (parmi d'autres) des régularités graphotactiques se répandrait et se stabiliserait rapidement. À suivre. Le second facteur d'influence relève des enseignements explicites mis en place pendant la scolarité. Ils s'appuient en général sur la formulation de règles suivie de séries plus ou moins longues et contrôlées d'exercices (copie, complètement, transpositions, etc.). Les performances évoluent en fonction de la pratique, passant d'une application lente, couteuse en attention et consciemment contrôlée à une relative automatisation permettant de gérer les situations les plus fréquentes (par exemple l'accord du verbe avec le sujet placé immédiatement avant lui). Toutefois, cette pratique s'accompagne de nouveaux apprentissages, implicites, de configurations : par exemple le fait que les 
verbes en français surviennent massivement immédiatement après les noms ou encore que certains verbes apparaissent très souvent à l'infinitif et plus rarement au participe passé (chercher, respecter, travailler...; Fayol \& Pacton, 2006). L'apparition d'erreurs nouvelles en est la conséquence: accord erroné des adjectifs (traités comme des verbes : les yeux fixent des poupées regardaient les passants), emploi erroné de l'infinitif (Ils ont chercher). De telles productions se révèlent à la fois rares et relativement systématiques : elles posent le problème de l'apprentissage de leur détection et de leur correction. De manière générale, l'étude des apprentissages présente un double intérêt. D'une part, elle conduit à considérer les évolutions décrites comme des produits de mécanismes qui doivent être identifiés et sur lesquels il est possible d'agir. D'autre part, elle permet d'établir des liens entre les enseignants ou les orthophonistes et les chercheurs, dans un sens comme dans l'autre: de la recherche vers son exploitation pour l'amélioration des performances; du constat des difficultés vers l'analyse des origines de celles-ci en vue d'optimiser les apprentissages et leur stabilité.

Pour rapide qu'il soit, ce tour d'horizon sur l'orthographe témoigne de la richesse et de la variété des recherches linguistiques et psycholinguistiques au cours de ces dernières années. Une période faste pour des analyses théoriques dont on aimerait désormais que les retombées dans l'usage et surtout dans l'enseignement, soient plus importantes.

\section{BIBLIOGRAPHIE}

ANIS, J. (dir.) (1983). « Le signifiant graphique ». Langue française, 59.

BADDELEY, S., JEJCIC, F. \& MARTINEZ, C. (éds) (2013). L'orthographe en quatre temps. $20^{e}$ anniversaire des Rectifications de l'orthographe de 1990. Enseignement, recherche et réforme, quelles convergences? Paris : Honoré Champion.

BROWN, G. D., \& LOOSEMORE, G. P. W. (1995). « Computational approaches to normal and impaired spelling ». In : Brown, G. D. A. \& Ellis, N. (eds), Handbook of spelling. Chichester: J. Wiley, p. 319-363.

CARAVOLAS, M. (2004). « Spelling development in alphabetic writing systems: A cross-linguistic perspective ». European Psychologist 9, p. 3-14.

CARAVOLAS, M., LERVÄG, A., MOUSIKOU, P., EFRIM, C., LITAVSKY, M., ONOCHIE-QUiNTANILLA, E., SALAS, N., SCHÖFFELOVA, M., DEFIOR, S., MIKULAJOVA, M., SEIDLOVA-MALOKOVA, \& HULME, C. (2013). « Common patterns of prediction of literacy development in different alphabetic orthographies ». Psychological Science 23 (6), p. 678-686.

CATACH, N. (1973a). « La structure de l'orthographe française ». La Recherche 4, 39, p. 949-956.

- (1973b). «Que faut-il entendre par système graphique du français ? ». Langue française 20, p. 30-44.

- (dir.) (1973c). « L'orthographe ». Langue française 20.

- (1978) [2003]. L'orthographe. Paris : Presses universitaires de France.

- (éd.) (1979). « Orthographe ». Pratiques 25.

- (1980). L'orthographe française. Traité théorique et pratique. Paris : Nathan. 
CERQUIGLINI, B. (1996). Le roman de l'orthographe. Au paradis des mots, avant la faute 1150-1694. Paris : Hatier [réédité en 2004 : La genèse de l'orthographe française, XII ${ }^{e}-\mathrm{XVII}^{e}$ siècle. Paris : Champion]. CHERVEL, A. (2006). Histoire de l'enseignement du français du XVII ${ }^{e}$ au XXe siècle. Paris : Retz. DANIELS, P. \& BRIGHT, W. (eds) (1996). The world's writing systems. Oxford : Oxford University Press. DEFRANCIS, J. (1984). The Chinese Language: Fact and Fantasy. Honolulu : University of Hawaï Press. - (1989). Visible Speech. The diverse oneness of writing systems. Honolulu : University of Hawaï Press. DUBOIS, J. (1965). Grammaire structurale du français. Nom et pronom. Paris : Larousse.

FAYOL, M. \& JAFFRÉ, J.-P. (2008). Orthographier. Paris : Presses universitaires de France.

- (2014). L'Orthographe. Paris : Presses universitaires de France.

FAYOL, M., HUPET, M. \& LARGY, P. (1999). « The acquisition of subject-verb agreement in written french. From novices to experts errors ». Reading and Writing 11, p. 153-174.

FAYOL, M., LARGY, P. \& LEMAIRE, P. (1994). « Subject- verb agreeement errors in French ». Quarterly Journal of Experimental Psychology 47A, p. 437-464.

FAYOL, M. \& PACTON, S. (2006). « L'accord du participe passé : entre compétition de procédures et récupération en mémoire ». Langue française 151, p. 59-73.

FAYOL, M., SAHLI, A. D.-I. \& SOUNY-BENCHIMOL, E. (2015). « L'apprentissage de l'orthographe des noms en situation de classe. Le cas des noms propres en histoire chez des élèves de première ».

Rééducation orthophonique 262, p. 161-174.

FRITH, U. (ed.) (1980). Cognitive Processes in Spelling. New York : Academic Press.

GRAINGER, J., DUFAU, S. \& ZIEGLER, J. C. (2016). « A vision of reading ». In : Trends in Cognitive Science, in press.

HENDERSON, L. (ed.) (1984). Orthographies and Reading. Londres : Lawrence Erlbaum.

HJELMSLeV, L. (1966) [1963]. Le langage. Trad. du danois par M. Olsen. Paris : Éditions de Minuit.

HO, C. S.-H. \& BRYANT, P. (1997). « Development of phonological awareness of Chinese children in Hong-Kong ». Journal of Psycholinguistic Research 26, 1, p. 109-126.

JAFFRÉ, J.-P. (2003). « Orthography: Overview ». In : Frawley, W.J. (ed.), Writing and Written language. The International Encyclopedia of Linguistics, vol. 4. Oxford: Oxford University Press, p. 386-390.

JAFFRÉ, J.-P. \& REUTER, Y. (éds) (1985). « Orthographe(s) ». Pratiques 46.

JOHNSON, S. (2005). Spelling trouble? Language, ideology and the reform of German orthography. Clevedon: Multilingual Matters.

JOSHI, R. M., \& AARON, P. G. (2006). Handbook of orthography and literacy. Mahwah: Lawrence Erlbaum.

KIM-RENAUD, Y.-K. (ed.) (1997). The Korean Alphabet. Its history and structure. Hawaï: University of Hawai'i Press.

LARGY, P., FAYOL, M. \& LEMAIRE, P. (1996). « The homophone effect in written French: The case of verb-noun inflection errors ». Language and Cognitive Processes 11, p. 217-255.

LUCCI, V. \& MILLET, A. (1994). L'orthographe de tous les jours. Paris : Honoré Champion.

MARTINET, C., VALDOIS, S. \& FAYOL, M. (2004). « Lexical orthographic knowledge develops from the beginning of reading acquisition ». Cognition 91, B11-B22. 
National Reading Panel (2000). Teaching children to read. Rockville: National Institute of Child Health and Human Development.

PACTON, S. \& FAYOL, M. (2000). « The impact of phonological cues on children's judgments of nonwords plausibility: the case of double letters ». Current Psychology Letter 1, p. 39-54.

- (2003). « Do French third and fifth graders use morphosyntactic rules when they spell adverbs and present participles?». Scientific Studies of Reading 7, p. 273-287.

PACTON, S., FAYOL, M. \& LÉTÉ, B. (2008). «L'intégration des connaissances lexicales et infralexicales dans l'apprentissage du lexique orthographique ». ANAE 96-97, p. 213-219.

PACTON, S., FAYOL, M. \& PERRUCHET, P. (2005). « Children's implicit learning of graphotactic and morphological regularities in French ». Child Development 76, p. 324-339.

PACTON, S., PERRUCHET, P., FAYOL, M. \& CLEEREMANS, A. (2001). « Implicit learning out of the lab.: The case of orthographic regularities ». Journal of Experimental Psychology: General 130, p. 401-426.

PELLAT, J.-C. (1988). « Indépendance ou interaction de l'écrit et de l'oral ? Recensement critique des définitions du graphème ». In : Catach, N. (éd.), Pour une théorie de la langue écrite. Paris : Éditions du CNRS, p. 133-146.

PERFETTI, C. A., RIEBEN, L. \& FAYOL, M. (eds) (1997). Learning to spell. Research, theory, and practice accross languages. Mahwah: LEA.

PUECH, C. \& ANIS, J. (1997). « Compte rendu de la table ronde sur l'autonomie de l'écriture ». In : Lapacherie, J.-G. (éd.), Propriétés de l'écriture. Pau : Publications de l'université de Pau, p. 79-87.

PULGRAM, E. (1951). «Phoneme and grapheme: a parallel ». Word 7, 1, p. 15-20.

RAPP, B. \& LIPKA, K. (2010). « The literate brain: The relationship between spelling and reading ». Journal of Cognitive Neuroscience 23, p. 1-18.

SAENGER, P. (1982). « Silent reading: its impact on late medieval script and society ». Viator 13, p. 363-414.

- (1989). «Physiologie de la lecture et séparation des mots ». Annales ESC 4, p. 9-952.

SAMPSON, G. (1985). Writing Systems. Londres : Hutchinson.

SAUSSURE, F. de (1972). Cours de linguistique générale. Paris : Payot.

SEBBA, M. (2007). Spelling and Society. Cambridge: Cambridge University Press.

- (2009). « Sociolinguistic approaches to writing systems research ». Writing Systems Research 1, 1, p. 35-49.

SEELEY, C. (1991). A history of writing in Japan. Leiden: E.J. Brill.

SEYMOUR, P. H., ARO, M. \& ERSKINE, J. M. (2003). « Foundation literacy acquisition in European orthographies ». British Journal of Psychology 94, p. 143-174.

SHARE, D. L. (2004). « Orthographic learning at a glance: On the time course and developmental onset of self-teaching ». Journal of Experimental Child Psychology 87, p. 267-298.

- (2008). « Orthographic learning, phonological recoding, and self-teaching ». In : KAIL, R. V. (ed.), Advances in child development and behavior. Amsterdam: Elsevier, p. 31-82.

SPRENGER-CHAROLLES, L., SIEGEL, L. S., BÉCHENNEC, D. \& SERNICLAES, W. (2003). « Development of phonological and orthographic processing in reading aloud, silent reading and in spelling: A five years longitudinal study ». Journal of Experimental Child Psychology 84, p. 194-217. 
TAINTURIER, M.-J. \& RAPP, B. (2001). « The spelling process ». In : Rapp, B. (éd.), The Handbook of cognitive neuropsychology. Hove: Psychology Press, p. 263-289.

TEMPLETON, S. \& BEAR, D. (eds.) (1992). Development of orthographic knowledge and the foundations of literacy. Hillsdale: LEA.

THÉVENIN, M. G, TOTEREAU, C., FAYOL, M. \& JAROUSSE, J. P. (1999). « L'apprentissage/enseignement de la morphologie écrite du nombre en français ». Revue française de pédagogie 126, p. 39-52.

TORGESEN, J. K. (2005). « Recent discoveries from research on remedial interventions for children with dyslexia ». In : Snowling, M. \& Hulme, C. (éds), The Science of Reading. Oxford: Blackwell, p. 521-537.

TOTEREAU, C., BARROUILLET, P. \& FAYOL, M. (1998). « Overgeneralizations of number inflections in the learning of written French: The case of nouns and verbs ». British Journal of Developmental Psychology 16, p. 447-464.

ULDALL, H. J. (1944). « Speech and writing ». Acta Linguistica 5, p. 11-16.

VACHEK, J. (1973). Written Language: General Problems and Problems of English. La Haye: Mouton.

ZIEGLER, J., BERTRAND, D., LÉTÉ, B., \& GRAINGER, J. (2014). « Orthographic and phonological contributions to reading development: Tracking developmental trajectories using masked priming ». Developmental Psychology 50 (4), p. 1026-1036.

ZIEGLER, J. C., BERTRAND, D., TOTH, D., CSÉPE, V., REIS, A., FAISCA, L., SAINE, N., LYYTINEN, H., VAESSEN, A. , \& BLOMERT, L. (2010). « Orthographic depth and its impact on universal predictors of reading: A cross-language investigation ». Psychological Science 21 (4), p. 551-559.

\section{NOTES}

1. Le terme chinois est utilisé ici par commodité. En République populaire de Chine, l'orthographe est en fait associée à une langue commune appelée putonghua, qui n'est pas la langue maternelle de tous les Chinois.

2. Véritable tempête dans un verre d'eau, la récente querelle sur une supposée "réforme » de l'orthographe, en apporte une nouvelle preuve.

3. Ce constat est d'une grande banalité (les «bons » en orthographe sont bons pour apprendre l'orthographe des mots nouveaux !), mais son explication reste mystérieuse à ce jour.

\section{RÉSUMÉS}

Ce texte propose un éclairage à la fois linguistique et psycholinguistique sur l'orthographe. Audelà de la spécificité inhérente, par définition, aux deux domaines, il illustre la complémentarité épistémologique qui s'est développée entre eux au cours des dernières décennies. La psycholinguistique a très souvent fait appel aux descriptions linguistiques pour élaborer ses hypothèses de travail et, de son côté, la linguistique s'est inspirée à maintes reprises des observations psycholinguistiques pour revisiter ses modèles théoriques. 
The $40^{\text {th }}$ birthday of the journal Pratiques offers us the opportunity to briefly review the situation of linguistic and psycholinguistic studies about orthography published during more or less the same period of time. In addition, we suggest some epistemological cooperation between the two domains. Psycholinguistics refers on linguistics to elaborate its working hypothesis and, on the other side, linguistics may draw its inspiration from psycholinguistics in order to reexamine its theoretical approaches.

\section{INDEX}

Mots-clés : orthographe, linguistique, psycholinguistique, acquisition

Keywords : orthography, linguistics, psycholinguistics, acquisition

\section{AUTEURS}

\section{MICHEL FAYOL}

Université Blaise Pascal Clermont-Ferrand, Centre national de la recherche scientifique, Lapsco, UMR 6024, F-63000, France

\section{JEAN-PIERRE JAFFRÉ}

Université Paris Ouest Nanterre La Défense, Centre national de la recherche scientifique, MoDyCo, UMR 7114, F-92001, France 